\title{
Maser effects in the recombination lines of Eta Carinae
}

1 Zulema Abraham, Augusto Damineli

Instituto Astronômico e Geofísico, Universidade de São Paulo, Brazil

Philippe Durouchoux

CE Saclay, France

Lars-Åke Nyman, and Felipe McAuliffe

ESO, La Silla, Chile

\begin{abstract}
Eta Car is a variable star surrounded by a cloud of gas and dust that absorbs a large part of its optical emission and that showed, in the past two hundred years, several events of mass ejection. It presents a 5.52 year period in the high excitation $\mathrm{He} \lambda 10830$ line intensity, indicating periodic changes in the ionizing radiation. Changes in the central velocity of optical lines, with the same periodicity, suggests the presence of a companion star, in a binary orbit. At radio frequencies, the region shows an optically thick bremsstrahlung spectrum, extending to millimeter and submillimeter wavelengths, and strong recombination lines at low quantum numbers showing maser effects. The flux densities, both from the continuum and recombination lines show a periodic behavior similar to that observed at optical wavelengths. We present observations of radio continuum and recombination lines at different millimeter wavelengths for several epochs. Based on these observations we modeled the emitting region as a thin disk and determined its temperature, electron density and velocity distributions using the radiation transfer equations in NLTE.
\end{abstract}

\section{Introduction}

Eta Car is a highly variable star. It presented several episodes of mass ejection, becoming one of the brightest stars in the sky during the 1837-1860 eruption. A 5.52 year period was found both in the equivalent widths of the high excitation lines and in the radial velocity of the broad $\mathrm{Pa} \gamma$ line, pointing to the existence of a binary system with masses of approximately $70 \mathrm{M}_{\odot}$ (Damineli 1996; Damineli et al. 2000). Variable radio emission was observed from $6 \mathrm{~cm}$ to $1 \mathrm{~mm}$ (Duncan, White, \& Lim 1997; Cox et al. 1995a; Abraham, \& Damineli 1999), showing the same periodicity. The radio continuum flux density increases with frequency, as expected from a partially optically thick region. VLBI observations with the

\footnotetext{
${ }^{1}$ Based on observations collected at the European Southern Observatory, La Silla, Chile
} 
Australia Telescope Compact Array (ATCA) showed, both in the continuum and in the $\mathrm{H} 91 \alpha$ recombination line, a disk like structure with its diameter decreasing with the total intensity (Duncan et al. 1997). While the $\mathrm{H} 91 \alpha$ recombination line has an HPW of the order of $500 \mathrm{~km} \mathrm{~s}^{-1}$, the higher frequency lines $\mathrm{H} 40 \alpha$ and $\mathrm{H} 30 \alpha$, are narrower and very intense, suggesting the presence of maser emission (Cox et al. 1995b). In this work we present observations of the continuum and recombination lines $\mathrm{H} 30 \alpha, \mathrm{H} 35 \alpha$ and $\mathrm{H} 40 \alpha$ at several epochs and propose a simple model for the region at the epoch of maximum intensity, that fits very well the amplitude and shape of all the recombination lines.

\section{Results}

We made observations with the SEST telescope at the frequencies of 99, 147 and $231 \mathrm{GHz}$, from which we obtained the continuum and the recombination lines $\mathrm{H} 40 \alpha, \mathrm{H} 35 \alpha$ and $\mathrm{H} 30 \alpha$, respectively. In Figure 1 we show the continuum spectrum and the ratio between the peak line intensity and the continuum for three epochs, Oct 99, Sept 00 and Jan 01 . We can see that the continuum flux density decreased at all frequencies since the first observation and that the the flux density at $147 \mathrm{GHz}$ had a sharp drop at the second epoch, producing changes also in the spectral shape. The line intensities changed in a different form, the $\mathrm{H} 30 \alpha$ line was stronger in Sep 00 than in the previous epoch, while the others were weaker. All of them had decrease by Jan 01. The line to continuum ratio increased in Sep 00 for all the lines and decreased again in Jan 01.

To try to understand the radio spectra and its time evolution, we modeled the emitting re gion as a thin edge on disk, of height $h$ and radius $R$, as suggested by the VLBI observations of Duncan et al. (1997) at lower frequencies.

We assumed constant temperature across the disk and an electron density distribution of the form $N_{e}=N_{0}\left(r_{\mathrm{in}} / r\right)^{a}$ for for $r_{\text {in }} \leq r \leq R$, where $r_{\text {in }}$ is the internal radius of the disk. Besides the thermal velocity, we introduced a constant turbulent velocity and a radial expansion velocity of the form $v(r)=$ $v_{0}+b r$.

We solved the equations of radiative transfer under NLTE conditions (see eg. Viener, Velee, \& Hughes 1979) and used the NLTE level populations calculated by Walmsley (1990). The radius of the disk was fixed as 2 ", as measured by Duncan et al. (1997) in the previous cycle, which corresponds to $0.024 \mathrm{pc}$ at the distance of $2.5 \mathrm{kpc}$.

For the Oct 99 data, the density profile turned out to be totally constrained by the continuum emission requirements. $N_{0}=8.8 \times 10^{6} \mathrm{~cm}^{-3}$ and $a=1$ gave the right continuum spectral index, the hight $h$ was chosen as $0^{\prime \prime} 36$ to fit the observed flux densities. The continuum intensity is not very sensitive to temperature, the ratio of peak line intensity to continuum, on the other hand, depends strongly not only on temperature but also on turbulent and expansion velocities (which widens the line, taking energy from the peak). The parameters, chosen to fit the lines are $T=8800 \mathrm{~K}, v_{\text {turb }}=20 \mathrm{~km} \mathrm{~s}^{-1}, v_{0}=-56 \mathrm{~km} \mathrm{~s}^{-1}$ and $b=10^{3} \mathrm{~km} \mathrm{~s}^{-1} \mathrm{pc}^{-1}$.

The line profiles obtained from our simple model can be seen in Figure 2 as filled circles, together with the observed recombination lines, represented by the solid line. The solutions of the transport equation under LTE assumption 

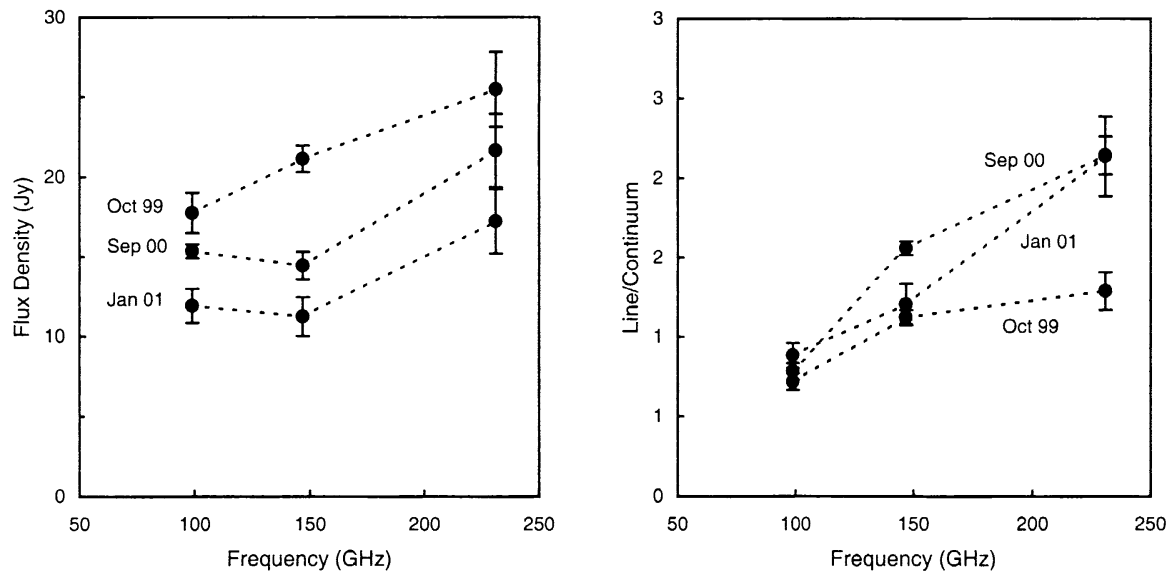

Figure 1. Left: continuum spectra of $\eta$ Car at three epochs, Oct 99, Sep 00 and Jan 01. Right: line peak and continuum flux densities for the $\mathrm{H} 40 \alpha, \mathrm{H} 35 \alpha$ and $\mathrm{H} 30 \alpha$, at the frequencies of 99,147 and $231 \mathrm{GHz}$, respectively

are shown as stars. We can see that the fitting of the NLTE model is very good, while the LTE calculations predict a line intensity ten times smaller that what is observed. Fitting the model to the other two epochs presented here is not so simple. In fact, due to the large change in the continuum spectral shape as a consequence of the sharp decrease in the $147 \mathrm{GHz}$ flux density, the emitting region cannot be represented by an electron density distribution in the form of a single power law and/or constant temperature, the same occurs with the recombination line intensities and profiles. This situation can be understood if the decrease in the continuum intensity were due to a decrease in the central ionizing radiation since the dense central region recombines and cools faster than the more diluted gas at the outer disk boundary.

\section{Conclusions}

A simple model of an expanding disk in NLTE, with constant temperature and a power law electron density distribution, can explain very well the continuum and $\mathrm{H} 30 \alpha, \mathrm{H} 35 \alpha$ and $\mathrm{H} 40 \alpha$ recombination lines observed in Oct 99 in Eta Car, when the continuum flux density was at its maximum. More complicated electron temperature and electron density distributions are necessary to explain the observations at later epochs, as expected from the decrease in the central ionizing flux, since recombination and cooling rates are different in the different regions of the disk.

Acknowledgments. This work was partially supported by the Brazilian agencies FAPESP, CNPq and FINEP. 

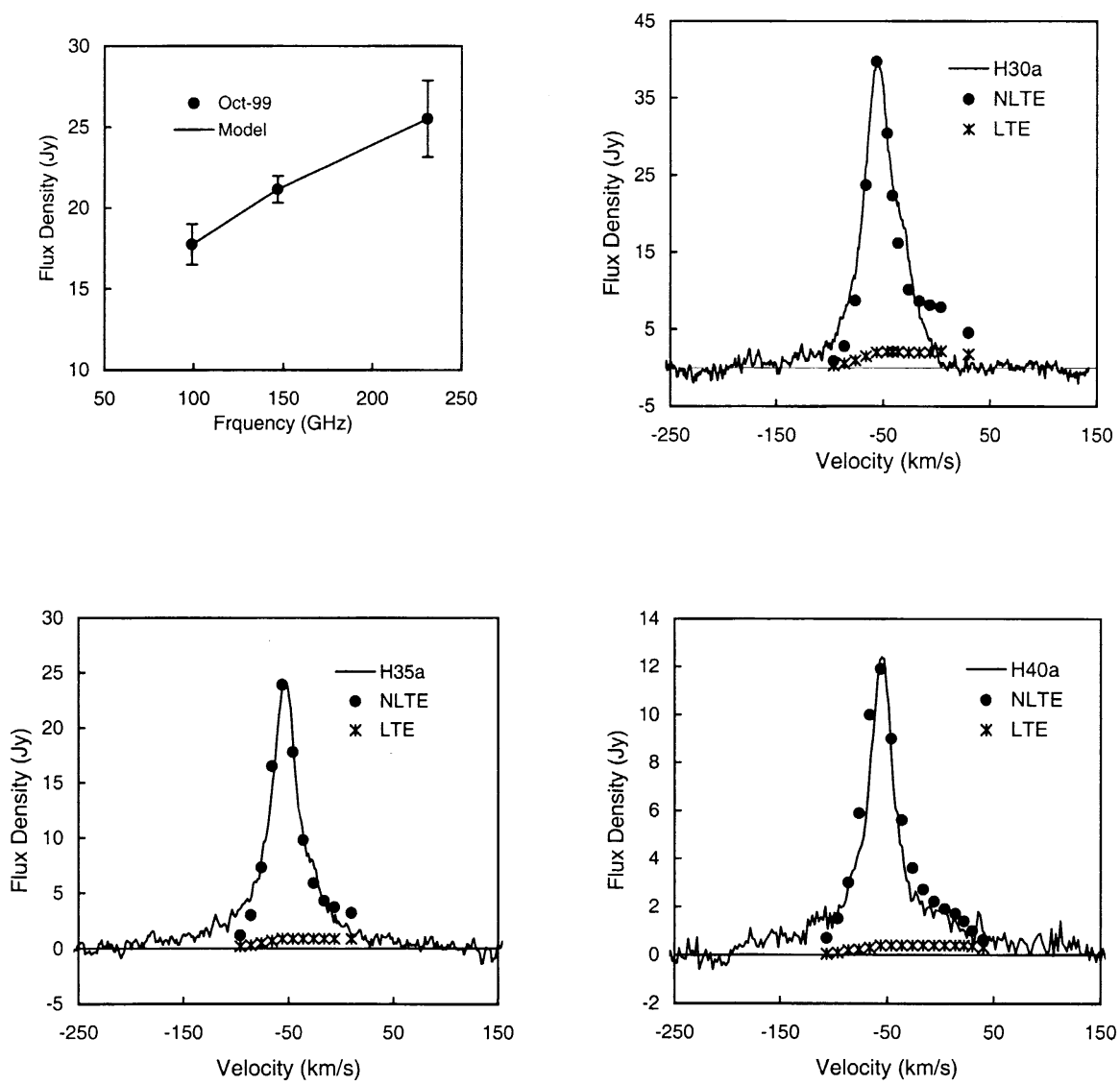

Figure 2. Continuum and recombination line fluxed densities in Oct 99 


\section{References}

Abraham, Z., Damineli, A. 1999, in ASP Conf. Ser. 179, $\eta$ Carinae at the Millennium, ed. J. A. Morse, R. Humphreys, \& A. Damineli (San Francisco: ASP), 263

Cox, P., Mezger, P. G., Sievers, A., et al. 1995a, A\&A, 297, 168

Cox, P., Martin-Pintado, J., Bachiller, R., et al. 1995b, A\&A, 295, L39 x Damineli, A. 1996, ApJ 460, L49

Damineli, A., Kaufer, A., Wolf, B. et al. 2000, ApJ, 528, L101

Duncan, R. A., White, S. M. \& Lim, J. 1997, MNRAS, 290, 680

Viner, M. R., Valee, J. P. \& Hughes, V. A. 1979, ApJ Supp, 39, 405

Walmsley C. M. 1990, A\&A, 82, 201

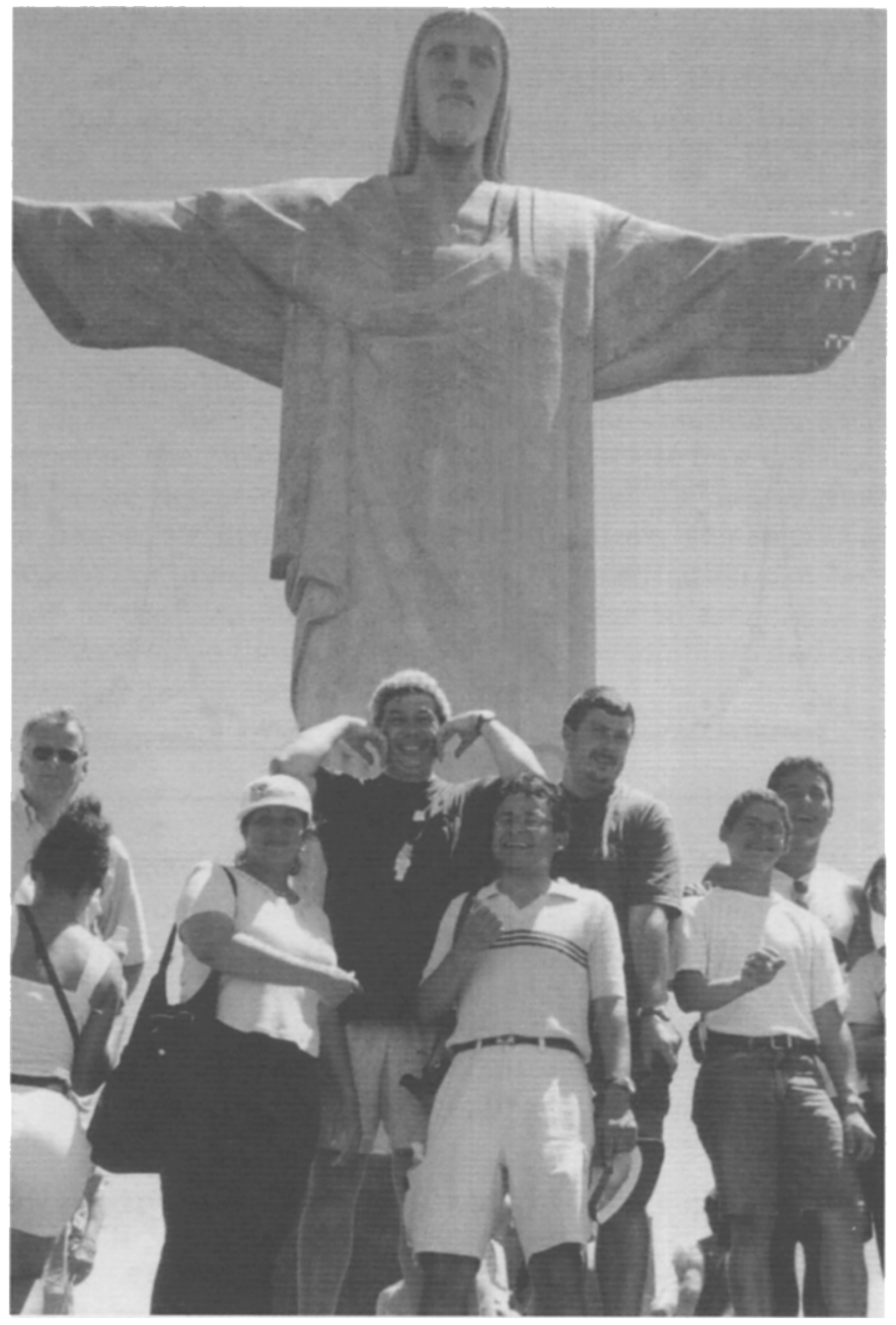

